\title{
¿POR QUÉ LOS JÓVENES SE DAN ATRACONES DE ALCOHOL LOS FINES DE SEMANA? ESTUDIO SOBRE CREENCIAS Y ACTITUDES RELACIONADAS CON ESTE PATRÓN DE CONSUMO Y DIFERENCIAS DE GÉNERO
}

\author{
RAFAEL BALLESTER ${ }^{1}$ Y M. DOLORES GIL ${ }^{2}$ \\ ${ }^{1}$ Universidad Jaime I de Castellón \\ ${ }^{2}$ Universidad de Valencia
}

\begin{abstract}
Resumen: Este trabajo expone los resultados de un estudio epidemiológico llevado a cabo en la Comunidad Valenciana (España) relativo al comportamiento y actitudes relacionadas con el consumo de alcohol de una muestra de 574 jóvenes de entre 18 y 24 años. Entre los principales resultados destaca que un $60 \%$ de los entrevistados consume habitualmente alcohol los fines de semana en dosis elevadas (el $20 \%$ bebe dos litros). Entre las motivaciones para su consumo destaca facilitar la diversión (83\%), las relaciones sociales $(77 \%)$ y evadirse de los problemas $(57 \%)$. El consumo de alcohol en el grupo de amigos es habitual para el $89 \%$. Aparecen importantes diferencias sexuales en el patrón de consumo (los chicos beben más que las chicas porque les facilita relacionarse), siendo el consumo de éstas mucho más moderado y asociándose con actitudes más adaptativas. La reacción ante las prohibiciones paternas, los aspectos sociales (facilita interacciones y presión grupal) y una falta de percepción de los riesgos asociados explican casi un 50\% de la varianza relativa a este patrón de consumo en fin de semana.
\end{abstract}

Palabras clave: Consumo de alcohol, atracón, jóvenes, diferencias de género.

\author{
Why young people binge-drink alcohol at weekends? A study about \\ beliefs and attitudes related to this consumption pattern and gender differences
}

\begin{abstract}
This work presents the results of an epidemiologic study done in the Comunidad Valenciana (Spain) about behaviours and attitudes related to alcohol consumption in a sample of 574 youngsters aged 18-24 years. One of the principal results points out that $60 \%$ of young people usually consume alcohol in large quantities during the weekend (20\% drinks two litres). Among the reasons to consume alcohol stand out diversion (83\%), to facilitate social interactions (77\%) and to evade problems (57\%). Alcohol consumption is habitual in the circle of friends for $89 \%$. Important gender differences appear in the pattern of alcohol consumption (boys drink more than girls because it helps social interaction), girls being more moderate and showing more adaptive attitudes. Opposition to paternal prohibitions, social aspects (such as easing interactions and group pressure) and the absence of risk perception explain almost $50 \%$ of the relative variance if this weekend drinking pattern.
\end{abstract}

Keywords: Alcohol consumption, binge-drinking, young people, gender differences.

\section{INTRODUCCIÓN}

El consumo y abuso de alcohol constituye uno de los mayores problemas de salud pública con que se enfrenta nuestra sociedad. En España, el alcohol es la sustancia psicoactiva de abu-

Recibido 6 mayo 2008; aceptado 19 junio 2008

Correspondencia: Rafael Ballester, Departamento de Psicología Básica, Clínica y Psicobiología, Universidad Jaime I de Castellón, Campus Riu Sec s/n, 12071 Castellón.

Correo-e: rballest@psb.uji.es so más extendida. Por los datos de que disponemos, según el Observatorio Español sobre Drogas (2007), el 61\% había consumido alcohol en los treinta días anteriores a la encuesta y el 15\% lo había hecho diariamente en este período. En un informe previo de este mismo organismo (2004) entre los 14 y los 18 años más del 30\% se habían emborrachado en el último mes. Según este estudio, el consumo de alcohol es mayor en hombres que en mujeres. Así, el porcentaje de hombres que en el último año habían realizado un consumo semanal de alco- 
hol era del 63\% frente al 33\% de mujeres. Esta misma diferencia se observa en la prevalencia de haberse emborrachado que era del $30 \%$ en hombres y del $13 \%$ en mujeres.

Atendiendo al patrón de consumo, en relación con la edad el estudio nacional encontró que en la población joven de entre 15 y 34 años está más extendido el consumo esporádico y la práctica de emborracharse (31\%) que en la población mayor entre 35 y 64 años (13\%), en la que es más frecuente el consumo diario (21\%) que en los jóvenes (6\%). En el estudio del Plan $\mathrm{Na}-$ cional sobre Drogas del año 2004, referido a datos del año anterior, el análisis de sectores de edad más concretos llevó a la conclusión de que el alcohol era la sustancia más consumida entre jóvenes de 14 a 18 años; que el 40\% de jóvenes de estas edades se había emborrachado en alguna ocasión y que sin embargo, apenas un 7,4\% tenía la percepción de consumir bastante o mucho alcohol.

Este patrón de consumo de alcohol en segmentos cada vez más jóvenes de la población es frecuente no sólo en nuestro país (Bueno et al., 1995; Mirón, Serrano, Godás y Rodríguez, 1997; Ballester, Gil y Guirado, 2000; Gil y Ballester, 2002) sino también en otros países europeos como Francia (Facy y Rabaud, 2006) o Estados Unidos (Lewinshon, Rohde y Seeley, 1996; Johnson, Stiffman, Hadley-Ives y Elze, 2001; Johnston, O'Malley y Bachman, 2006).

Se trata, por tanto, de un grave problema que afecta a una buena parte de la sociedad en su mayoría joven y de forma diferencial en función del género. Se caracteriza por un patrón específico de atracón durante los fines de semana asociado a la diversión y que se caracteriza por una escasa percepción de los riesgos asociados. Sin embargo, los riesgos para la salud son numerosos. En primer lugar, las intoxicaciones etílicas provocan un elevado número de muertes en accidentes de tráfico (Hoyert, Heron, Murphy y Kung, 2006; Pickrell, 2006; Eaton, Kann y Kinchen, 2006; Shope, Raghunathan y Patil, 2003). En segundo lugar la escasa percepción del riesgo a la que induce el alcohol también se asocia con un mayor número de conductas sexuales de riesgo, por ejemplo, para el contagio del VIH (MacDonald, MacDonald, Zanna y Fong, 2000; Collins, Ellickson, Orlando y Klein, 2005). Así, en un es- tudio de Bonomo et al. (2001) con jóvenes de 17 años se observó que el 70\% bebían alcohol, que uno de cada diez jóvenes decía tener relaciones sexuales bajo los efectos del alcohol y que todos éstos reconocían que su consumo afectaba a sus relaciones sexuales, tanto desde el punto de vista de disfunciones sexuales como de prácticas inseguras. Más allá de estas dos causas de mortalidad de la juventud tan importantes podemos encontrar otros muchos ámbitos donde el abuso del alcohol genera numerosos riesgos personales e individuales tales como el comportamiento agresivo y violento (Komro et al., 1999; Sierra, Gutiérrez y Delgado, 2008), el abandono académico (Muthén y Muthén, 2000), el ajuste psicológico de los adolescentes (Oliva, Jiménez, Parra y Sánchez, 2008), el juego patológico (Fernández-Montalvo, Landa y López-Goñi, 2005), la delincuencia (Bui, Ellickson y Bell, 2000), el desarrollo de trastornos de personalidad (Fernández-Montalvo, Landa, López-Goñi, Lorea y Zarzuela, 2002) y el desarrollo de un trastorno por abuso de sustancias (D'Amico, Ellickson, Collins, Martino y Klein, 2005).

A la luz de estos datos es obvia la urgencia de desarrollar programas de prevención realistas que partan de un conocimiento exhaustivo de los factores que explican el inicio y el mantenimiento de este patrón de consumo de los jóvenes. Como señalan Coleman y Cater (2006) dada la extensión del problema puede ser más eficaz una estrategia de reducción de daños que una que promueva la abstinencia. Así, es preciso centrarse en los jóvenes como grupo de especial riesgo máxime cuando los datos indican que el riesgo de inicio de un trastorno por abuso de alcohol tiene su cima en la transición de la adolescencia a la juventud (Chung, Martin y Winters, 2006).

En este sentido, la investigación sobre los posibles predictores de ese consumo abusivo de alcohol se ha centrado fundamentalmente en el clima y funcionamiento familiar (Rowe y Liddle, 2006), así como en las influencias de los compañeros (Fergusson y Horwood, 1996) aspectos que analizaremos en este trabajo.

Finalmente, la investigación sobre el patrón de consumo de alcohol de adolescentes y jóvenes no resulta sencilla, ya que la mayoría de jóvenes que consumen alcohol y otras drogas no buscan ayuda como consecuencia del estigma asociado 
a estas conductas y el temor respecto a la confidencialidad de la información. Los profesionales de los servicios de asistencia se quejan de que los jóvenes no hablan de este tema pero éstos manifiestan que aunque desearían hacerlo, tienen miedo a ser juzgados (Stern, Meredith, Gholson, Gore y D'Amico, 2007). Otros estudios como el de Van Hook et al. (2007) que también han denunciado la dificultad que se tiene en los servicios de atención primaria para identificar casos de abuso de alcohol en jóvenes, han indicado como principales inconvenientes la falta de tiempo y de entrenamiento de los profesionales, además de la dificultad que añaden los padres que no están dispuestos a abandonar la sala para facilitar una conversación confidencial.

Así pues la estrategia más sencilla y eficaz de detección de problemas asociados al abuso del alcohol puede ser preguntar directamente a los jóvenes en contextos no clínicos donde la garantía de confidencialidad sea máxima. Esta es la estrategia que se ha seguido en el presente trabajo cuyo objetivo es estudiar el patrón de consumo de alcohol entre los jóvenes analizando las actitudes y creencias que sustentan dicho patrón con vistas a la elaboración de planes de prevención primaria y secundaria en la población general.

\section{MÉTODO}

\section{Participantes}

La muestra se compone de 574 jóvenes de edades comprendidas entre 18 y 24 años con la siguiente distribución: $27,9 \%$ de 18 años; $16,9 \%$ de 19 años; $14,6 \%$ de 20 años; $13,6 \%$ de 21 años; $9,9 \%$ de 22 años; $7 \%$ de 23 años y 10,1\% de 24 años. Por sexo, el 67,9\% de la muestra eran chicas y el $32,1 \%$, chicos. Para la obtención de la muestra se contactó con distintos centros educativos y asociaciones juveniles de ámbito rural $(27 \%)$ y urbano $(73 \%)$ de las tres provincias de la Comunidad Valenciana.

\section{Instrumentos de evaluación}

En este estudio epidemiológico se han utilizado algunos de los items del CIACS-III (Cues- tionario de Información, Actitudes y Comportamientos relacionados con la Salud-III) de Ballester y Gil, el cual forma parte de un estudio epidemiológico más amplio en el que se exploran distintas conductas de salud. Este instrumento tiene una fiabilidad evaluada a través del alfa de Cronbach (consistencia interna) de 0,94 y una fiabilidad test-retest de 0,88 (Ballester y Gil, 2007). Para el presente trabajo se han utilizado los 16 items relativos al consumo de alcohol (ver Anexo 1).

Como se puede apreciar en el Anexo 1, cuatro items del cuestionario aluden al comportamiento de los adolescentes respecto al consumo de alcohol, ocho se refieren a sus actitudes, uno habla del consumo de sus amigos, tres del consumo de los padres y dos de las actitudes de los padres respecto al consumo de los hijos. Las opciones de respuesta difieren en función del contenido del ítem. En algunos se utilizó una escala Likert (desde 1: nunca o nada de acuerdo hasta 4: siempre/habitualmente o totalmente de acuerdo). De este tipo son los items 9, 10, $11,55,56,57,58,59,60,61,62,63,78,144$ y 145 (utilizamos la numeración original del cuestionario completo). En los items 141, 142, 143, las respuestas son abiertas.

\section{Procedimiento}

Para reunir la muestra se seleccionaron distintos centros de Formación Profesional, institutos y centros universitarios, así como asociaciones y colectivos juveniles de distintas localidades tanto rurales como urbanas de las provincias de Castellón, Valencia y Alicante. Tras solicitar los permisos prescriptivos se procedió a la administración colectiva del CIACS-III de manera anónima, confidencial y voluntaria.

\section{RESULTADOS}

\section{Análisis descriptivos de la muestra global}

Los análisis descriptivos muestran que más de la mitad de los sujetos encuestados $(60 \%)$ consume habitualmente alcohol durante los fines de semana. El 44\% de estos sujetos consu- 
me entre medio litro y un litro y el $20 \%$ entre uno y dos litros. El 25\% consume menos de un litro pero sólo un 1,7\% no bebe nada de alcohol. En cuanto al tipo de bebida, la mitad de los sujetos dice tomar bebidas tipo «vermut» entre las que se incluye el Martini o el Malibú, y casi un 20\%, bebidas fuertes (en torno a $40^{\circ}$ ), que incluyen el whisky, el vodka, el tequila, la ginebra o el ron. Aproximadamente un 30\% de los encuestados consume generalmente bebidas que hemos catalogado como «suaves» (entre 5 y $15^{\circ}$ ) y entre las que podemos encontrar la cerveza, el vino, la sidra y el cava. Apenas un 4\% consume bebidas dulces de $30^{\circ}$ o más como el Ponche, el anís o el moscatel.

En cuanto a información y actitudes, el 30\% de los sujetos percibe una elevada presión grupal para consumir alcohol. El 60\% de los jóvenes piensa que el alcohol genera dependencia y que no es fácil dejarlo pero sólo el 33\% piensa que no tiene ningún efecto positivo sobre la salud. El 52\% cree que puede ser beneficioso en algún sentido y el $15 \%$ está bastante o totalmente de acuerdo en que el consumo de alcohol durante las comidas es saludable. Hay que señalar que más del $80 \%$ considera que tiene suficiente información acerca de los efectos del alcohol.

En este segmento de edad sólo el $8 \%$ bebe para aparentar ser más mayor, a diferencia de los datos superiores encontrados en la población adolescente (Ballester, Gil y Guirado, 2000). Las motivaciones más importantes entre los jóvenes son otras. Así, el 57\% considera que beber sirve para evadirse de los problemas, el $77 \%$ piensa que beber facilita las relaciones sociales, el $14 \%$ que ayuda a resolver sus problemas y el mayor porcentaje (83\%) considera que beber facilita la diversión. Parece, por tanto, que los jóvenes conceden un importante papel al alcohol en el ocio ya que les facilita la diversión y las interacciones sociales.

Con respecto a creencias relativas al consumo de alcohol y deterioro de la salud, el $87 \%$ cree que sólo es perjudicial si se consume diariamente; el $85 \%$, sólo si se consume durante muchos años; el 74\% piensa que sólo es perjudicial si se llega a la embriaguez o se mezclan distintas bebidas; el $67 \%$, si se mezcla con otras drogas; el $63 \%$, si se tolera poco; y el $51 \%$, si no se ha comido previamente.
Dada la importancia que el modelado y los aspectos de relación pueden tener en el inicio y mantenimiento del consumo de alcohol, se analizaron también los patrones sociales de consumo de su entorno. El 89\% indicó que el consumo de alcohol entre sus amigos era algo habitual. El $81 \%$ de los jóvenes informan que sus padres toman vino en las comidas, el $77 \%$ cerveza, el $31 \%$ whisky y el $32,5 \%$ carajillo (café con coñac). Estos altos porcentajes contrastan con el $87,5 \%$ de jóvenes que indican que en su casa está muy mal visto el consumo de alcohol. Hay que destacar, por otro lado, que el $12 \%$ de jóvenes encuestados indica que en sus casas no está mal considerado el consumo de alcohol.

\section{Análisis diferenciales en función del sexo}

Para el análisis de las posibles diferencias existentes en función del sexo se realizaron pruebas $t$ de Student. En cuanto al comportamiento de los jóvenes en relación con el alcohol (items 144 y 10) (ver Tabla 1) aparecen diferencias estadísticamente significativas en función del sexo en la frecuencia de consumo de alcohol (ítem 144) encontrando una media de frecuencia de consumo mayor en chicos que en chicas (los chicos consumen en mayor medida alcohol todos o casi todos los días y las chicas lo hacen en mayor medida durante los fines de semana o únicamente en celebraciones). En esta misma línea encontramos que la media de frecuencia de borracheras es mayor en varones que en las chicas (ítem 10).

En el siguiente grupo de items relacionados con las actitudes y creencias de los jóvenes respecto al consumo de alcohol (items 56, 57, 58, 61, 62 y 63) los resultados muestran actitudes menos saludables entre los varones comparados con las mujeres (ver Tabla 1). Así, los hombres muestran estar más de acuerdo que las mujeres con la idea de que «si eres de una pandilla es normal tomar lo mismo que los demás» (ítem 56). También ocurre esto con la idea de que el alcohol no produce dependencia (ítem 57), significativamente más compartida por los chicos que por las chicas y también con la creencia de que beber alcohol en las comidas es saludable (ítem 58). Las diferencias persisten en la misma 
Tabla 1. Diferencias entre las variables en función del sexo (pruebas t de Student)

\begin{tabular}{|c|c|c|c|c|c|c|}
\hline \multirow[b]{2}{*}{$N .^{o}$} & \multirow[b]{2}{*}{ Item } & \multicolumn{2}{|c|}{ Mujeres } & \multicolumn{2}{|c|}{ Hombres } & \multirow{2}{*}{$t$} \\
\hline & & Media & $D T$ & Media & $D T$ & \\
\hline 10 & Me he emborrachado & 2,12 & 0,71 & 2,36 & 0,86 & $-3,49 * * *$ \\
\hline 56 & $\begin{array}{l}\text { Si eres de una pandilla es normal tomar lo mismo que los } \\
\text { demás }\end{array}$ & 1,37 & 0,71 & 1,53 & 0,78 & $-2,43 * *$ \\
\hline 57 & $\begin{array}{l}\text { El alcohol no engancha, cualquiera puede dejarlo cuan- } \\
\text { do quiera }\end{array}$ & 1,50 & 0,80 & 1,92 & 1,04 & $-5,27 * * *$ \\
\hline 58 & Beber alcohol en las comidas es saludable & 1,80 & 0,75 & 1,99 & 0,82 & $-2,82 * *$ \\
\hline 59 & $\begin{array}{l}\text { Tengo bastante información acerca de los efectos del al- } \\
\text { cohol }\end{array}$ & 3,24 & 0,83 & 3,25 & 0,92 & $-0,10$ \\
\hline \multirow[t]{2}{*}{61} & Cuando bebo parezco más adulto & 1,07 & 0,33 & 1,21 & 0,57 & $-3,57 * * *$ \\
\hline & Cuando bebo me resulta más fácil: & & & & & \\
\hline 62.1 & Evadirme de los problemas & 2,02 & 1,08 & 1,95 & 1,04 & 0,59 \\
\hline 62.2 & Relacionarme con la gente & 2,66 & 1,12 & 2,40 & 1,09 & $2,48 * *$ \\
\hline 62.3 & Resolver mis problemas & 1,17 & 0,56 & 1,27 & 0,62 & $-1,69$ \\
\hline 62.4 & Divertirme & 2,65 & 1,02 & 2,71 & 1,05 & $-0,60$ \\
\hline 63 & El alcohol deteriora la salud siempre & 2,98 & 1,16 & 2,64 & 1,22 & $2,88 * *$ \\
\hline \multirow[t]{2}{*}{60} & En mi grupo de amigos es habitual el consumo de alcohol & 2,83 & 1,04 & 3,07 & 0,98 & $2,52 * *$ \\
\hline & Mis padres toman: & & & & & \\
\hline 11.1 & Vino en las comidas & 2,40 & 1,01 & 2,39 & 1,01 & 0,03 \\
\hline 11.2 & Cerveza & 2,05 & 0,84 & 2,25 & 0,95 & $-2,26^{*}$ \\
\hline 11.3 & Whisky o cubata & 1,34 & 0,58 & 1,45 & 0,65 & $-1,65$ \\
\hline 11.4 & Carajillo & 1,48 & 0,80 & 1,41 & 0,71 & 0,79 \\
\hline 55 & En mi casa está muy mal visto el consumo de alcohol & 2,64 & 0,95 & 2,43 & 0,97 & $2,45^{* *}$ \\
\hline 144.1 & ¿Consumes alcohol todos los días? & 1,02 & 0,79 & 1,46 & 0,49 & $1,92 *$ \\
\hline 144.2 & ¿Consumes alcohol casi todos los días? & 1,04 & 0,82 & 1,35 & 0,82 & 1,65 \\
\hline 144.3 & ¿Consumes alcohol los fines de semana? & 2,03 & 1,02 & 1,47 & 0,74 & $-2,03^{*}$ \\
\hline 144.4 & $\begin{array}{l}\text { ¿Consumes alcohol sólo en las celebraciones u oca- } \\
\text { siones importantes? }\end{array}$ & 2,60 & 0,80 & 1,20 & 0,93 & $-2,89 * *$ \\
\hline 145 & Cuando eras pequeño tus padres te daban alcohol & 1,40 & 0,58 & 1,60 & 0,72 & $-0,71$ \\
\hline
\end{tabular}

$* p<0,05 ; * * p<0,01 ; * * * p<0,001$

línea en el ítem 61 «cuando bebo parezco más adulto», creencia más informada por los chicos que por las chicas (ver Tabla 1).

El ítem 62 que incluye cuatro subvariables explora las posibles razones por las que los jóvenes deciden beber. No encontramos diferencias significativas entre el grado de acuerdo de chicos y chicas en que al beber les resulta más fácil evadirse de los problemas, resolverlos o divertirse, pero sí en que cuando beben les resulta más fácil relacionarse con la gente, si bien en este caso la media mayor es la perteneciente a las chicas (ver Tabla 1). El ítem 63 muestra diferencias significativas entre hombres y mujeres al considerar éstas en mayor medida que aquéllos que el alcohol deteriora la salud siempre.

En un tercer bloque hemos reunido los items que exploran el consumo por parte de los amigos, de los padres y también las actitudes de éstos respecto al consumo de los hijos (items 60, $55,11,145)$. En primer lugar, hay diferencias significativas en el grado de acuerdo de chicas 
y chicos respecto al ítem 60 («En mi grupo de amigos es habitual el consumo de alcohol») siendo superior la media relativa a los chicos que la de las chicas (ver Tabla 1). Lo mismo ocurre en el ítem 55 en el que se pregunta si en su hogar está mal considerado el consumo de alcohol. Parece que en el caso de las chicas el alcohol está peor considerado por los padres que en el de los chicos. Por el contrario, en el caso del ítem 11 referido al consumo de alcohol por parte de los padres, las diferencias entre las medias no alcanzan el nivel de significación estadística. Y, por último, en el ítem 145 se recoge el comportamiento de los padres en relación con el consumo de alcohol del joven durante su infancia pero las diferencias no alcanzan el grado de significación estadística.

\section{Correlaciones entre variables}

Para indagar la posible relación existente entre variables se realizaron análisis de correlación de Pearson. En primer lugar, vemos que consumir durante los fines de semana y emborracharse correlacionan significativamente entre sí $(r=0,38, p<0,01)$ (ver Tabla 2). En segundo lugar, el patrón de consumo de fines de semana correlaciona positiva y significativa- mente sobre todo con el consumo habitual de alcohol en el grupo de amigos $(r=0,35, p<0,01)$ $\mathrm{y}$ también pero en menor medida con la idea de que si eres de una pandilla es normal tomar lo mismo que los demás $(r=0,11, p<0,01)$ y con el consumo paterno de vino $(r=0,11, p<0,05)$, cerveza $(r=0,10, p<0,05)$ y whisky $(r=0,12$, $p<0,05)$. En tercer lugar, en cuanto a la embriaguez, la principal correlación también ese da con el consumo habitual de alcohol en el grupo de amigos $(r=0,37, p<0,01)$, seguida con el mismo resultado $(r=0,16, p<0,01)$ por la idea de que es normal tomar lo mismo que los otros de la pandilla y el consumo de cerveza por parte de los padres, pero no de vino ni whisky.

\section{Análisis de regresión}

Finalmente, a partir de las correlaciones encontradas se ha llevado a cabo un análisis de regresión múltiple (método de pasos sucesivos) para ver qué variables de las que han mostrado una relación significativa (variables independientes) pueden explicar el consumo de alcohol en fin de semana (variable dependiente). Como se puede observar en la Tabla 3, con tres variables se explica el $46 \%$ de la varianza. Es-

Tabla 2. Correlaciones significativas entre variables relacionadas con el consumo de alcohol durante los fines de semana

\begin{tabular}{clcc}
\hline & & Consumo fin semana (144.3) & Emborracharse (10) \\
\hline 144.3 & Consumo fin semana & \\
10 & Emborracharse & $0,38^{* *}$ & \\
9 & Padres prohíben & $0,32^{* *}$ & $0,27^{* *}$ \\
11.1 & Padres-vino & $0,11^{*}$ & - \\
11.2 & Padres-cerveza & $0,10^{*}$ & $0,16^{* *}$ \\
11.3 & Padres-whisky & $0,12^{*}$ & - \\
56 & Pandilla & $0,11^{* *}$ & $0,16^{* *}$ \\
60 & Habitual en grupo & $0,35^{* *}$ & $0,37^{* *}$ \\
63.3 & Deteriora salud sólo emborracharse & $0,38^{* *}$ & $0,30^{* *}$ \\
78 & Probar distintas drogas & $0,34^{* *}$ & $0,33^{* *}$ \\
\hline
\end{tabular}

Nota: Ítem 144.3. ¿Consumes alcohol los fines de semana?; Ítem 10: Me he emborrachado; Ítem 9: Mis padres no me permiten beber alcohol; Ítem 11: Mis padres toman: (a) Vino en las comidas, (b) Cerveza, (c) Whisky o cubata; Ítem 56: Si eres de una pandilla es normal tomar lo mismo que los demás; Ítem 60: En mi grupo de amigos es habitual el consumo de alcohol; Ítem 63.3: El alcohol sólo deteriora la salud si llegas a emborracharte; Ítem 78: No es malo probar diferentes drogas. $* p<0,05 ; * * p<0,01$ 
tas variables son: «El alcohol deteriora la salud sólo si llegas a emborracharte» (ítem 63.3), «Mis padres no me permiten beber alcohol» (ítem 9) $\mathrm{y}$ «No es malo probar diferentes drogas» (ítem 78). Al parecer el consumo excesivo de alcohol en fin de semana es característico de jóvenes que no asumen los peligros del alcohol (minimizan sus consecuencias y no acaban de percibir el riesgo de deterioro de la salud porque no llegan a etiquetar su estado como de embriaguez) ni tampoco los peligros del hecho de probar diferentes drogas y reaccionan ante la prohibición paterna consumiendo excesivamente, es decir, escasa percepción del riesgo y conducta contestataria.

Tabla 3. Análisis de regresión (pasos sucesivos) para explicar el consumo de alcohol durante los fines de semana

\begin{tabular}{ccccc}
\hline Paso & $\begin{array}{c}\text { Variable } \\
\text { independiente }\end{array}$ & Beta & $\mathrm{R}_{+}^{2}$ & $\mathrm{~F}(29)$ \\
\hline 1 & V63.3 & 0,41 & 0,22 & \\
2 & V9 & 0,55 & 0,36 & \\
3 & V78 & 0,37 & 0,46 & $7,45^{* * *}$ \\
\hline
\end{tabular}

Nota: V63.3:«El alcohol deteriora la salud si llegas a emborracharte»; V9:«Mis padres no me permiten beber alcohol»; V78:«No es malo probar diferentes drogas». $* * * p<0,001$.

\section{DISCUSIÓN}

El primer aspecto a destacar observando los resultados de nuestro estudio es que se confirma la tendencia al aumento del consumo de alcohol, en primer lugar comparado con décadas anteriores (Czechowicz, 1991) y en segundo lugar, con el del segmento anterior de edad, en la línea de los resultados obtenidos por el Observatorio Español sobre Drogas (2003, 2004, 2007). En el presente estudio hemos encontrado que sólo el 1,7\% de los jóvenes encuestados no bebe nada. Se trata de un porcentaje mucho más reducido que el que obtuvimos en un trabajo previo en sujetos de edades comprendidas entre los 14 y los 18 años (9.5\%) (Ballester, Gil y Guirado, 2000). Por otro parte, más de la mitad de los encuestados consume durante los fi- nes de semana cantidades que les llevan a emborracharse. Es decir, que se constata que no sólo ha aumentado el número de jóvenes que beben sino que se ha modificado el patrón de consumo, resultados coincidentes con numerosos trabajos, tanto de nuestro entorno más próximo (Mateos, Páramo, Carrera y RodríguezLópez, 2002; Parker y Egginton, 2002; Observatorio Español sobre drogas, 2003, 2004, 2007), como del resto de Europa (Facy y Rabaud, 2006) y USA (Johnston, O'Malley y Bachman, 2006; Tucker, Orlando y Ellickson, 2003).

Por lo que respecta a las actitudes y creencias en relación con el consumo de alcohol más de la mitad de los jóvenes $(60 \%)$ en nuestro estudio está de acuerdo en que el alcohol genera dependencia y que no es fácil dejarlo, consideración que ha aumentado en comparación con el segmento anterior de edad (14 a 18 años) donde esto sólo lo opinaba el 45\% (Ballester, Gil y Guirado, 2000). El motivo podría radicar en que con la edad los sujetos van acumulando experiencia en relación con las consecuencias negativas del consumo de alcohol al tiempo que experimentan sus primeras dificultades para reducir su consumo. En este sentido la información ambigua que a menudo nos ofrecen los medios de comunicación de masas con respecto a los efectos beneficiosos del consumo de alcohol en determinadas condiciones pueden contribuir a generar confusión y aunque la mayoría dice tener suficiente información con respecto a los efectos del alcohol, un buen porcentaje se muestra indeciso con respecto a los beneficios del mismo. A este respecto, Sulkunen (1998) alerta de que la investigación social y cultural relativa al alcohol es una ventana de dos direcciones, que nos permite mirar la sociedad a través del alcohol y el alcohol a través de la sociedad siendo ambas visiones necesarias para comprender las cuestiones implícitas que sustentan los patrones desadaptativos de consumo generalizados en nuestra sociedad en los últimos años.

Otro aspecto a comentar es la importancia del consumo de alcohol en el hogar como variable relacionada con la ingesta frecuente e incontrolada del mismo por parte del joven, coincidiendo con los resultados obtenidos por Rowe y Liddle (2006). Más de un $80 \%$ de nuestros encuestados han crecido viendo beber alcohol en 
sus hogares de forma habitual, algo que también sucede en otros hábitos como el consumo de tabaco (Bermúdez y Contreras, 2008). Es este un dato especialmente preocupante cuando se atiende a los resultados de estudios como el llevado a cabo en Suecia por Lundborg (2002). Este investigador encontró que tener padres que consumen alcohol y toleran su ingesta aumenta la frecuencia de consumo de cerveza, vino y licor, la intensidad del consumo de estas bebidas y la probabilidad de emborracharse. Este dato podemos interpretarlo teniendo en cuenta de nuevo la ambigüedad de los mensajes recibidos por los jóvenes. Así, en nuestro estudio en el $87 \%$ de los hogares de los entrevistados está mal visto el consumo de alcohol, pero en el $80 \%$ es consumido por los padres. Esta doble moral, la vieja consigna de «haz lo que yo digo pero no lo que yo hago» puede justificar la escasa repercusión de la educación relativa al alcohol en relación con su consumo posterior. Efectivamente el modelado familiar tiene una importancia crucial en el desarrollo y consolidación de conductas adictivas en la edad adulta (Gotham, Sher y Word, 2003; Chung, Martin y Winters, 2006). Por otro lado, algunos estudios indican que algunos problemas graves de abuso de alcohol en la familia salen a la luz cuando se pregunta a los adolescentes y jóvenes por el consumo de los padres en el hogar (Padilla y Rodríguez-Franco, 2003).

Por otra parte hemos constatado la existencia de diferencias entre chicos y chicas en cuanto al consumo y creencias relacionadas con el alcohol. Estos resultados van en la línea de los obtenidos en varios estudios (Nystrom, 1993; Kushner y Sher, 1993; Fergusson y Horwood, 1996) si bien la tendencia futura, como ocurrió hace algunos años con el consumo del tabaco (Bermúdez y Contreras, 2008), va hacia un incremento del consumo por parte de ellas (Mateos et al., 2002).

Por lo que se refiere a los coeficientes de correlación obtenidos entre las distintas variables analizadas en este estudio podemos ver que existe cierta cohesión entre un determinado grupo de creencias y comportamientos ya que correlacionan significativamente entre sí, lo que nos hace pensar que se alimentan o justifican mutuamente. Así ya hemos visto que la fre- cuencia del consumo de alcohol, el emborracharse, el consumo por parte de los padres, el consumo de los amigos y la idea de que es normal tomar lo mismo que éstos correlacionan significativamente, en la línea de otros estudios como el de Lundborg (2002) o Gotham, Sher y Wood (2003).

Por último, el análisis de regresión nos muestra que este patrón de consumo en fin de semana se explica bastante bien (elevado porcentaje de la varianza explicado) a partir de cuestiones relativas al grado de conocimiento de los jóvenes sobre los efectos del alcohol (cuándo el alcohol afecta la salud) y las actitudes de los padres hacia el consumo de los hijos.

Como conclusiones más destacadas de nuestro estudio podemos subrayar, de un lado, la coincidencia con otros estudios epidemiológicos (Comisión Clínica para el Plan Nacional de Drogas, 2007) en cuanto al creciente consumo tanto en frecuencia como en intensidad de alcohol en el segmento de jóvenes con edades comprendidas entre los 18 y los 24 años, y de otro, la relación existente entre el modelado del consumo por parte de amigos y padres con la gestación de patrones desadaptativos de consumo. Las creencias de los jóvenes con respecto a las consecuencias del consumo de alcohol para la salud son acordes con su comportamiento y, seguramente podemos afirmar que actitudes y comportamientos se alimentan mutuamente. En la base de ambos, comportamiento y creencias, está el estándar socialmente validado que marca el modo en que se debe desarrollar el ocio a esas edades. Existe una presión grupal enorme (recordemos que en el $89 \%$ de los casos sus amigos consumen alcohol), a menudo no percibida, que dicta el modo «socialmente correcto» de divertirse. Hay que estar desinhibido a nivel social, evadirse de los problemas y estar contento para estar bien visto en el grupo, todo lo cual se consigue en buena parte con ayuda del alcohol. En este sentido el miedo a la evaluación negativa por parte de los demás (Gallego, Botella, Quero, Baños y García-Palacios, 2007) especialmente en la adolescencia y el consumo de alcohol pueden constituir una pareja peligrosa. Mientras se mantengan estas exigencias, el consumo abusivo de alcohol (o el de otras drogas con efectos semejantes) se man- 
tendrá impermeable a los programas de prevención centrados en el cambio de actitudes y/o de comportamientos. Los programas de prevención primaria y secundaria deberían tener en cuenta estos resultados a la hora de ser diseñados e implementados.

\section{REFERENCIAS}

Ballester, R., y Gil, M.D. (2007). Cuestionario de información, actitudes y comportamientos relacionados con la salud (CIACS-I, II y III). V World Congress of Behavioural and Cognitive Therapies. Barcelona, 11-14 julio.

Ballester, R., Gil, M.D., y Guirado, M.C. (2000). Comportamientos y actitudes relacionados con el consumo de alcohol en adolescentes de 15 a 17 años. Análisis y Modificación de Conducta, 110, 855-898.

Bermúdez, J., y Contreras, A. (2008). Predictores psicosociales del consumo de tabaco en adolescentes: Extensiones de la teoría de la conducta planificada. Revista de Psicopatología y Psicología Clínica, 13, 175-186.

Bonomo, Y., Coffey, C., Wolfe, R., Lynskey, M., Bowes, G., y Patton, G. (2001). Adverse outcomes of alcohol use in adolescents. Addiction, 96, 1485-96.

Bueno, F.J., Gavidia, V., Gómez, J., Salazar, A., Sieres, J., y Valderrama, J.C. (1995). Hábitos de salud en la juventud de Valencia. Valencia: Concejalía de salud y consumo del Ayuntamiento de Valencia.

Bui, K.V.T., Ellickson, P.L., y Bell, R.M. (2000). Crosslagged relationships among adolescent problem drug use, delinquent behavior, and emotional distress, Journal of Drug Issues, 30, 283-303.

Chung, T., Martin, C.S., y Winters, K.C. (2006). Diagnosis, course and assessment of alcohol abuse and dependence in adolescents. En M. Galanter, Alcohol problems in adolescents and young adults: Epidemiology, neurobiology, prevention, and treatment (pp. 5-27). New York: Springer Science.

Coleman, L.M., y Cater, S. (2006). Pathways for practice and policy to reduce adolescent alcohol abuse: Interpretations from a qualitative study. International Journal of Adolescent Medicine and Health, 18, 37-41.

Collins, R.L., Ellickson, P.L., Orlando, M., y Klein, D.J. (2005). Isolating the nexus of substance use, violence and sexual risk for HIV infection among young adults in the United States. AIDS and Behavior, 9, 73-87.

Comisión Clínica de la Delegación del Gobierno para el Plan Nacional sobre Drogas (2007). Informe sobre alcohol: Febrero 2007. Madrid: Ministerio de Sanidad y Consumo.

Czechowicz, D. (1991). Adolescent alcohol and drug addiction and its consequences: an overview. En N.S.
Miller, Comprehensive handbook of drug and alcohol addiction (pp. 205-210). New York: Marcel Dekker.

D’Amico, E.J., Ellickson, P.L., Collins, R.L., Martino, S.C., y Klein, D.J. (2005). Processes linking adolescent problems to substance use problems in late young adulthood. Journal of Studies on Alcohol, 66, 766-775.

Eaton, D.K., Kann, L., y Kinchen, S. (2006). Youth Risk Behavior Surveillance-United States, 2005, MMWR Surveill Summ 55, 1-108.

Facy, F., y Rabaud, M. (2006). Towards the prevention of alcohol abuse. Drugs: Education, Prevention and Policy, 13, $139-149$.

Fergusson, D.M., y Horwood, L.J. (1996). The role of adolescent peer affiliations in the continuity between childhood behavioral adjustment and juvenile offending. Journal of Abnormal Child Psychology, 24, 205-221.

Fernández-Montalvo, J., Landa, N., López-Goñi, J.J., Lorea, I., y Zarzuela, A. (2002). Trastornos de personalidad en alcohólicos: Un estudio descriptivo. Revista de Psicopatología y Psicología Clínica, 7, 217-225.

Fernández-Montalvo, J., Landa, N., y López-Goñi, J.J. (2005). Prevalencia del juego patológico en el alcoholismo: un estudio exploratorio. Revista de Psicopatología y Psicología Clínica, 10, 125-134.

Gallego, M.J., Botella, C., Quero, S., Baños, R.M., y García-Palacios, A. (2007). Propiedades psicométricas de la Escala de Miedo a la Evaluación Negativa (versión breve) en muestra clínica. Revista de Psicopatología y Psicología Clínica, 12, 163-176.

Gil, M.D., y Ballester, R. (2002). Inicio temprano de consumo de alcohol entre niños de 9 a 14 años. Análisis y Modificación de Conducta, 28, 165-211.

Gotham, H.J., Sher, K.J., y Wood, P.K. (2003). Alcohol involvement and developmental task completion during young adulthood. Journal of Studies on Alcohol, 64, $32-42$.

Hoyert, D., Heron, M., Murphy, S., y Kung, H.C. (2006). Deaths: final data for 2003. Natl Vital Stat Rep, 54, 1-120.

Oliva, A., Jiménez, J.M., Parra, A., y Sánchez, I. (2008). Acontecimientos vitales estresantes, resiliencia y ajuste adolescente. Revista de Psicopatología y Psicología Clínica, 13, 56-62.

Johnson, S.D., Stiffman, A., Hadley-Ives, E., y Elze, D. (2001). An analysis of stressors and co-morbid mental health problems that contribute to youths' paths to substance-specific services. Journal of Behavioral Health Services \& Research, 4, 412-426.

Johnston, L., O’Malley, P., y Bachman, J. (2006). Monitoring the Future: National Results on Adolescent Drug Use, Overview of Key Findings, 2005. Bethesda: National Institute on Drug Abuse.

Komro, K.A., Williams, C.L., Forster, J.L., Perry, C.L., Farbakhsh, K., y Stigler, M.H. (1999). The relationship 
between adolescent alcohol use and delinquent and violent behavior. Journal of Child \& Adolescent Substance Abuse, 9, 13-28.

Kushner, M.G., y Sher. K.J. (1993). Comorbidity of alcohol and anxiety disorders among college students: effects of gender and family history of alcoholism. $A d$ dictive Behaviors, 18, 543-552.

Lewinsohn, P.M., Rohde, P., y Seeley, J.R. (1996). Alcohol consumption in high school adolescents: frequency of use and dimensional structure of associated problems. Addiction, 91, 375-390.

Lundborg, P. (2002). Young people and alcohol: an econometric analysis. Addiction, 97, 1573-1582.

MacDonald, T.K., MacDonald, G., Zanna, M.P., y Fong, G.T. (2000). Alcohol, sexual arousal, and intentions to use condoms in young men: applying alcohol myopia theory to risky sexual behavior. Health Psychology, 19, 290-298.

Mateos, R., Paramo, M., Carrera, I., y Rodríguez-López, A. (2002). Alcohol consumption in a southern European region (Galicia-Spain). Substance Use and Misuse, 37, 1957-1976.

Mirón, L., Serrano, G., Godás, A., y Rodríguez, D. (1997). Conducta antisocial y consumo de drogas en adolescentes españoles. Análisis y Modificación de Conducta, 88, 255-282.

Muthen, B.O., y Muthen, L.K. (2000). The development of heavy drinking and alcohol-related problems from ages 18 to 37 in a U.S. national sample, Journal of Studies on Alcohol, 61, 290-300.

Naranjo, C.A., y Bremner, K.E. (1993). Bahavioural correlates of alcohol intoxication. Addiction, 88, 31-41.

Nyström, M. (1993). Alcohol use, drinking patterns and indicators of heavy drinking in Finnish university students. Helsinki: Yliopistopaino.

Observatorio Español sobre Drogas (2003; 2004; 2007). Informe anual de la Delegación del Gobierno para el Plan Nacional sobre Drogas. Madrid: Ministerio de Sanidad y Consumo.
Padilla, E.M., y Rodríguez-Franco, L. (2003). Detección de problemas relacionados con el alcohol en la familia a través de los hijos. Revista de Psicopatología y Psicología Clínica, 8, 139-152.

Parker, H., y Egginton, R. (2002). Adolescent recreational alcohol and drugs careers gone wrong: developing a strategy for reducing risks and harms. International Journal of Drug Policy, 13, 419-432.

Pickrell, T. (2006). Driver alcohol involvement in fatal crashes by age group and vehicle type. National Highway Traffic Safety Administration's National Center for Statistics and Analysis. NHTSA Research Note DOT HS 810598.

Rowe, C.L., y Liddle, H.A. (2006). Family-based treatment development for adolescent alcohol abuse. International Journal of Adolescent Medicine and Health, $18,43-51$.

Shope, J.T., Raghunathan, T.E., y Patil, S.M. (2003). Examining trajectories of adolescent risk factors as predictors of subsequent high-risks driving behavior. Journal of Adolescent Health, 32, 214-224.

Sierra, J.C., Gutiérrez, J.R., y Delgado, J.C. (2008). Primer estudio psicométrico de la versión española del Aggresive Behavior Inventory (ASBI). Revista de Psicopatología y Psicología Clínica, 13, 21-31.

Stern, S.A., Meredith, L.S., Gholson, J., Gore, P., y D'Amico, E.J. (2007). Project CHAT: A brief motivational substance abuse intervention for teens in primary care. Journal of Substance Abuse Treatment, 32, 153-165.

Sulkunen, P. (1998). Images and realities of alcohol. Addiction, 93, 1305-1312.

Tucker, J.S., Orlando, M., y Ellickson, P.L. (2003). Patterns and correlates of binge drinking trajectories from early adolescence to young adulthood. Health Psychology, 22, 79-87.

Van Hook, S., Harris, S.K., Brooks, T., Carey, P., Kossack, R., Kulig, J., y Knight, J.R. (2007). The «Six T's»: Barriers to screening teens for substance abuse in primary care. Journal of Adolescent Health 40, 456-461. 
Anexo 1: Items pertenecientes al CIACS-III relacionados con el consumo de alcohol.

9. Mis padres no me permiten beber alcohol.

10. Me he emborrachado

11. Mis padres toman:

11.1. Vino en las comidas

11.2. Cerveza

11.3. Whisky o cubata

11.4. Carajillo después de comer

55. En mi casa está muy mal visto el consumo de alcohol

56. Si eres de una pandilla es normal tomar lo mismo que los demás

57. El alcohol no engancha, cualquiera puede dejarlo cuando quiera

58. Beber alcohol en las comidas es saludable

59. Tengo bastante información acerca de los efectos del alcohol

60. En mi grupo de amigos es habitual el consumo de alcohol

61. Cuando bebo parezco más adulto

62. Cuando bebo me resulta más fácil:

62.1. Evadirme de los problemas

62.2. Relacionarme con la gente

62.3. Resolver mis problemas

62.4. Divertirme

63. El alcohol deteriora la salud:

63.1. Siempre

63.2. Sólo si no has comido

63.3. Si llegas a emborracharte

63.4. Si consumes muchos años

63.5. Si consumes todos los días

63.6. Sólo si mezclas distintas bebidas

63.7. Sólo si lo mezclas con otras drogas

63.8. Si lo toleras poco

78. No es malo probar diferentes drogas.

141. ¿Te has emborrachado alguna vez? ¿A qué edad fue la primera?

142. ¿Cuánta y qué tipo de bebida alcohólica tomas si vas de fiesta?

143. ¿Alguien en tu familia bebe excesivamente? ¿Quién?

144. Indica el grado de acuerdo con las siguientes afirmaciones:

144.1 ¿Consumes alcohol todos los días?

144.2 ¿Consumes alcohol casi todos los días?

144.3 ¿Consumes alcohol los fines de semana?

144.4. ¿Consumes alcohol sólo en las celebraciones u ocasiones importantes?

145. Cuando eras pequeño tus padres te daban alcohol:

145.1. Nunca

145.2. En las celebraciones

145.3. Cuando estabas resfriado

145.4. Para entrar en calor cuando habías cogido frío

145.5. Cuando perdías el apetito 